Rev. Elev. Méd. Vét. Pays trop., 1976, 29 (2) : 115-117.

\title{
Note sur deux cas d'haemobartonellose féline observés au Tchad
}

\author{
J.-L. DELAITRE ( $\left.{ }^{*}\right)$
}

\begin{abstract}
RÉSUME
Deux chats atteints de cette affection, signalée pour la première fois au Tchad, ont été guéris par traitement aux antibiotiques majeurs. L'état très alarmant d'un des deux malades a même justifié une transfusion sanguine dont l'effet semble avoir largement contribué à sa guérison.
\end{abstract}

"L'anémie infectieuse » des chats, entité clinique due à Haemobartonella felis, parasite des globules rouges, est caractérisée par une anémie importante et de l'hyperthermie ; elle est diagnostiquée de plus en plus souvent en France.

Il nous a été permis d'en observer et d'en suivre deux cas au cours de cette année à la Clinique Vétérinaire de N'Djamena (Tchad).

\section{OBSERVATIONS CLINIQUES}

\section{Premier cas}

Chat mâle entier, genre siamois, âgé d'un an.

\section{Symptômes}

L'animal est amené à la clinique le 2 décembre 1972 : il est prostré, ne mange plus depuis 3 jours.

L'amaigrissement est très important, la déshydratation est moyenne (les yeux sont légèrement enfoncés dans les orbites et il y a procidence du corps clignotant).

L'anémie des muqueuses est très marquée : il sera difficile de ponctionner du sang à l'extrémité de l'oreille et les intraveineuses seront délicates.

(*) Institut d'Enseignement Zootechnique et Vétérinaire d'Afrique Centrale, N'Djamena, Tchad.
La rate est grosse et facilement palpable.

L'hyperthermie est importante : $40,1^{\circ} \mathrm{C}$.

Pas de signes digestifs ni pulmonaires sinon une légère polypnée.

Cliniquement l'on a donc affaire à un " chat blanc » qui semble se laisser mourir.

\section{Diagnostic}

Seuls les examens hématologiques, d'ailleurs trẻs simples, peuvent permettre de poser le diagnostic de façon certaine, les symptômes étant loin d'être pathognomoniques.

- La numération globulaire, qui doit être réalisée presque systématiquement dans ce genre d'affection pour éliminer la leucopénie, révèle une anémie intense : 2500000 érythrocytes $/ \mathrm{mm}^{3}$; une leucocytose élevée : 23000 globules blancs $/ \mathrm{mm}^{3}$, ce qui permet d'éliminer rapidement la panleucopénie au point de vue diagnostic.

- Des frottis sanguins ont été réalisés : ils sont colorés selon la méthode classique de MAYGRUNWALD et GIEMSA ; ils permettent d'établir la formule sanguine suivante :
Neutrophiles : 60
Eosinophiles : 5
Lymphocytes : 32
Monocytes : 1
Plasmocytes : 2

ce qui est pratiquement normal chez le chat. 
Par contre, de très nombreux érythroblastes révélateurs de l'anémie sont dénombrés et surtout l'agent causal de la maladie est mis en évidence de façon très nette; il s'agit d'Haemobartonella felis. On constate, par ailleurs, l'anisocytose et la polychromatophilie des hématies.

Les nombreux éléments très nettement colorés sont le plus souvent intracellulaires mais certains se retrouvent libres dans le plasma. La plupart des parasites sont sphériques et leur diamètre peut être estimé entre 0,2 et 0,3 microns; certains sont plus ou moins rectangulaires et situés à la périphérie des érythrocytes (parfois même ils semblent comme accolés à la membrane des globules).

\section{Traitement}

1 er jour :

Une réhydratation est immédiatement mise en œuvre pour soutenir l'animal qui est complètement dénutri.

Pour ce faire, on lui injecte sous la peau :

$30 \mathrm{ml}$ de sérum glucosé isotonique,

$30 \mathrm{ml}$ de sérum salé isotonique,

$10 \mathrm{ml}$ de chophytol.

Puis, à la suite de la lecture des lames, un traitement spécifique est entrepris.

Ce traitement spécifique est effectué à l'aide d'un mélange antibiotique à base de

Tétracycline : $50 \mathrm{mg}$

Chloramphénicol (1): $200 \mathrm{mg}$

Prednisolone : $10 \mathrm{mg}$

Vitamine $B_{12}: 500 \mu \mathrm{g}$

(sous forme de préparation injectée en intramusculaire).

Ce traitement antibiotique sera poursuivi le plus longtemps possible : au moins une dizaine de jours pour éviter les rechutes.

$2^{\mathrm{e}}$ jour :

- l'état général est bien meilleur : la température est subnormale $\left(38,7^{\circ} \mathrm{C}\right)$, l'appétit reprend ;

- le traitement antibiotique est poursuivi pendant 10 jours et il conviendrait certainement de le continuer plus longuement mais cela est impossible pour des raisons pratiques ;

(1) «Chloramfrécortyl » de Vétoquinol.
- l'état général est soutenu par des injections quotidiennes de

sérum glucosé : $20 \mathrm{ml}$, sérum salé : $20 \mathrm{ml}$, chophytol : $10 \mathrm{ml}$;

- un traitement buccal anti-anémique à base de «Lysine Egic» est mis en cuvre pendant 2 semaines.

\section{Devenir de l'animal}

Le chat en question a été traité en décembre 1972 ; depuis, il a été revu plusieurs fois : des frottis et des numérations ont été effectués (en janvier et en mars 1973) qui n'ont rien révélé d'anormal mais cela ne permet pas de conclure à une guérison car cette note est écrite 6 mois seulement après le premier accident.

\section{Deuxième cas}

Chat mâle castré, batard de siamois et de local, âgé de 6 ans.

Symptômes

Amené la première fois à la clinique le 20 février 1973 avec les mêmes symptômes que le premier, mais plus accusés : l'animal est prostré depuis 6 jours, ne mange plus, il a considérablement maigri aux dires du propriétaire ; il est très déshydraté et très anémié ; la température est de $40,3^{\circ} \mathrm{C}$. Bref, le tableau clinique est sombre.

\section{Diagnostic}

La numération globulaire qui donne moins de 2000000 d'érythrocytes, plus de 20000 leucocytes,

permet d'éliminer la leucopénie et l'examen des frottis met en évidence, là encore de façon très nette, les parasites en très grand nombre : dans ce deuxième cas d'ailleurs, on observe de très nombreux éléments libres dans le plasma.

\section{Traitement}

Pendant 3 jours, le même traitement antibiotique et de réhydratation est mis en auvre, mais - s'il contribue à faire descendre la température - il ne semble pas agir sur l'état général de l'animal qui reste très faible et sans appétit.

Une transfusion sanguine est alors tentée : $40 \mathrm{ml}$ de sang sont prélevés par voie intracardiaque sur un chat amené pour sacrification ; ils 
sont mélangés dans la seringue à $5 \mathrm{ml}$ de mélange anticoagulant $\mathrm{ACD}$ (acide citrique, citrate trisodique, glucose) utilisé pour le même usage en médecine humaine et réinjectés avec la même seringue dans la saphène interne en moins de $3 \mathrm{mn}$.

Cette transfusion se révèlera salvatrice pour l'animal qui est véritablement « remis sur pieds".

A cause des difficultés de réalisation (chat donneur difficile à trouver) et pour éviter un choc éventuel, cette opération n'a pas été renouvelée.

Le traitement antibiotique ne sera poursuivi que pendant une semaine : c'est peut-être ce qui explique la rechute rapide que nous aurons à observer sur cet animal.

\section{Devenir de l'animal}

En effet, le 23 mars 1973, soit un mois après le premier accident, l'animal est de nouveau présenté à la clinique. Il présente les mêmes symptômes, l'anémie est moins importante : 3500000 érythrocytes, mais aucun parasite n'a pu être mis en évidence sur les frottis qui ont pourtant été renouvelés.

Toutefois, un traitement antibiotique asso- ciant tétracycline et chloramphénicol est instauré pendant 10 jours.

A ce jour, c'est-à-dire 3 mois après, l'animał se porte bien mais rien ne laisse préjuger de son avenir.

\section{QUELQUES REMARQUES}

"L'anémie infectieuse" des chats domestiques semble donc assez fréquente au Tchad: pour en poser le diagnostic, il faut s'attacher à réaliser systématiquement la numération sanguine (rouge et blanche) ainsi que la formule sur des frottis qui permettront la mise en évidence des parasites qui sont très facilement colorables.

La fréquence des parasites externes au Tchad, en particulier des tiques, peut être un argument en faveur de l'hypothèse souvent émise par les auteurs anglo-saxons (1) selon laquelle la maladie serait transmise naturellement par des arthropodes piqueurs.

La transfusion sanguine doit jouer un grand rôle dans le traitement de l'anémie infectieuse qui est à base d'antibiotiques majeurs (tétracycline, chloramphénicol). Mais il faut insister sur la durée du traitement qui doit être longue (peut-être 15 jours) pour éviter des rechutes éventuelles.

\section{SUMMARY}

Note about two cases of cat haemobartonelosis observed in Chad

Two cats attacked by this disease, noted for the first time in Chad, recovered with antibiotic treatment.

The alarming state of one of these two animals necessitated a blood transfusion the effect of which seems to have favoured the cure.

\section{RESUMEN}

\section{Nota sobre dos casos de hemobartonelosis del gato observados en Chad}

Dos gatos atacados por esta enfermedad señalada por la primera vez en Chad han curado después de un tratamiento con los antibioticos mayores. El estado muy alarmante de uno de los enfermos ha necesitado incluso una transfusión sanguinea cuyo efecto parece haber favorecido la curación.

\section{BIBLIOGRAPHIE}

1. EDWARDS (F. B.). A new blood parasite in british cats. Vet. Rec., 1960, 72 (22) : 439.

2. GROUlade (P.). Clinique canine. Tome I. Paris, Maloine, 1965.

3. WILKINSON (G. T.). Feline infectious anemia. Ver. Rec., 1969, 84 (13) : 331-333. 